\title{
Analysis of an eHealth app: Privacy, Security and Usability
}

\author{
Ryan Alturki ${ }^{1}$, Mohammed J. AlGhamdi ${ }^{5}$ \\ Department of Information Sciences \\ Umm Al-Qura University, Makkah, Saudi Arabia
}

\author{
Valerie $\mathrm{Gay}^{2}$ \\ School of Electrical and Data Engineering \\ University of Technology, Sydney, Australia
}

Abstract-Obesity and overweight are considered a health threat globally. Saudi Arabia is a country that has a high percentage of people suffering from obesity. These people can be helped to lose weight through the usage of mobile apps as these apps can collect users' personal information. These collected data is used to provide precise and personalized weight loss advices. However, weight loss apps must be user friendly, provide data security and user privacy protection. In this paper, we analyze the usability, security, and privacy of a weight loss app. Our main aim to clarify the data privacy and security procedure and test the usability level of the new Arabic weight loss app 'Akser Waznk' that is developed considering the social and cultural norms of Saudi users.

\section{Keywords-Obesity; usability; data security; privacy; app}

\section{INTRODUCTION}

Obesity means storing extra energy in the shape of fat [1]. It can lead to many health problems, such as diabetes and cardiovascular issues [2,3]. More than one-third of the Saudi population suffers from obesity [4]. The unique features within weight loss apps can motivate users to improve their behavior and start to lose weight $[5,6]$. Though, users want such apps to have a high level of usability and can provide data privacy and security [7]. Therefore, there is a dire need of such apps that are easy to use and preserve user privacy along with security. In this paper, we have analyzed the usability for the Arabic weight loss app "Akser Waznk" which is designed for Saudi obese users. In the beginning, we discuss the procedures that need to be implemented in order to provide data privacy and security protection. In the following section, the usability testing attributes, procedure, participations information and the list of tasks that are performed within the usability testing were presented. The following section provides the results of the usability attributes that were tested. After that, we discuss the usability issues which were highlighted by the potential users of the app and explains how such issues can be addressed to improve the usability level of the app. Finally, the paper ends with the conclusion.

\author{
Nabeela Awan ${ }^{3}$, Mehwish Kundi ${ }^{6}$ \\ Department of Computer Science \\ Abdul Wali Khan University Mardan, Mardan, Pakistan
}

\author{
Mohammad Alshehri ${ }^{4}$ \\ Department of Computer Science \\ Taif University, Taif, Saudi Arabia
}

\section{DATA PRIVACY AND SECURITY}

Akser Waznk collects sensitive personal information from users to provide them with precise and personalized weight loss advices. Information that can identify persons or their natural is defined as personal information likewise for the mobile information [8,9]. Therefore, the app applies number of standards which are based on the European Commission's Code of Conduct on privacy for mHealth apps [10] and the EU's General Data Protection Regulation (GDPR) [8] to assure data privacy and security protection. These standards contain users' consent, minimizing required data and purpose limitation. When users want to start using the app, they are provided with a set of questions and clarifications regarding the method and goals of the app before they agree to provide the requested personal information, such as weight and height. Such information is used to calculate users' BMI value, ideal weight and provide precise diet plans. Moreover, the app asks for users' permission to access and retrieve data from iPhone Health feature to count the daily number of walking steps and measure distance. Beside this, users can read a detailed privacy policy that is written in a simple Arabic language explaining the reason for collecting data, indicating privacy statements and providing users with the app's developer contacting details. Akser Waznk's privacy policy can easily be found via the setting option in the app or the Apple Store. Furthermore, the app applies encryption of the data to protect users' information. Algorithms is used to convert readable texts to unreadable texts or codes to generate an advanced level of data security [11]. Such cipher text cannot be decrypted without an encryption key that is only available via the authorised parties [12]. Two kind of data will be protected, in transit and at rest data. The in transit data refers to the data moving between locations, such as when a user inputs data to the app and then the data is moved to the app's server or database as well as the structure of messaging system between a user and mobile will be in secured channel [13]. At rest data is the data that is saved in a cloud or database and not moving between locations [12]. Both kind of data are considered by Akser Waznk and the encryption is applied for them. Moreover, the app notifications are applied trust metrics to ensure the data privacy and security among users and mobiles $[14,15]$. 


\section{USABILITY TESTING}

\section{A. Usability Attributes}

Effectiveness, efficiency and satisfaction are the key aspects of usability according to the International Standards Organisation (ISO) 9241-11 [16]. Other attributes, for example, learnability, memorability, cognitive load and errors are related to both apps' effectiveness and efficiency and recommend being considered [17, 18]. While each of the aforementioned attributes help to measure the efficiency and effectiveness of apps, they do this from different points of view. A lesser error rate signifies that apps are effective as users can perform more takes within a short period of time without errors. Also, when apps have better learnability and memorability users can more accurately perform more tasks even though they might not regularly use apps and therefore both effectiveness and efficiency are met. Moreover, when apps have a better cognitive load level, users will be able to perform several actions while using apps, for example, a user might drink coffee and speak to friends while using an app. Thus, apps become easier to use. Each of the above attributes, therefore, enhance the usability and user satisfaction.

\section{B. Procedure and Participations}

The usability testing for Akser Waznk is performed following the same techniques and procedures that were used in our previous work to test the usability level of two Arabic weight loss apps (Twazon and Aded Surat) [19, 20]. This is because Akser Waznk is developed based on the results of the previous work and by following the same roles, we can then compare this result with the previous results and indicate whether or not the level of usability is improved. Earlier usability guides indicate that around $80 \%$ of usability issues within a product can be detected by having 5 participants within a usability testing experiment. Moreover, other usability guides believe that $90 \%$ of usability issues within a product also can be detected by having 10 participants within a usability testing experiment [21-23]. Based on this and in order to cover all the possible issues that may occur while performing the usability testing for the app, the potential users group comprised of 26 obese Saudi Arabians. There were 13 men and 13 women in the group. Table I shows the potential users' information.

\section{The Tasks}

Each participant within the usability testing of the app is asked to perform 14 tasks. The aim from this is to fully explore the app which can help to detect any usability issue. The tasks are:

1) Make an account and fill out all the information.

2) Add the activity, "running for 20 minutes".

3) Add a Bed time snack meal.

4) Find out how many calories in that meal.

5) Send the word "Hi" to Naser.

6) Adds a reminder to the day at $1.30 \mathrm{pm}$.

7) Adjust the target number of daily steps to 10 steps.

8) Change the theme of the app.

9) Find when "almaghrib" prayer time.
10) Add that you "drank 2 bottles of water that have the size of $350 \mathrm{ml}$ ".

11) Read the example of the nutrition label.

12) Change your weight to $80.5 \mathrm{~kg}$.

13)Donate to Makkah charity.

14)Review what you have done for this day.

\section{TABLE I. POTENTIAL USER'S INFORMATION}

\begin{tabular}{|c|c|c|c|}
\hline User & Gender & Age Group & Type of iPhone \\
\hline 1 & Male & 35 to 44 & iPhone $\mathrm{x}$ \\
\hline 2 & Male & 25 to 34 & iPhone 7 \\
\hline 3 & Female & 25 to 34 & iPhone $\mathrm{x}$ \\
\hline 4 & Female & 45 to 54 & iPhone 6s \\
\hline 5 & Female & 25 to 34 & iPhone $\mathrm{x}$ \\
\hline 6 & Female & Prefers not to say & iPhone 7 \\
\hline 7 & Female & 25 to 34 & iPhone 7 plus \\
\hline 8 & Female & 25 to 34 & iPhone 6s \\
\hline 9 & Female & Prefers not to say & iPhone 7 \\
\hline 10 & Female & Prefers not to say & iPhone 7 \\
\hline 11 & Male & 18 to 24 & iPhone 6 \\
\hline 12 & Female & 18 to 24 & iPhone 7 plus \\
\hline 13 & Female & 45 to 54 & iPhone 6s plus \\
\hline 14 & Male & 25 to 34 & iPhone $\mathrm{x}$ \\
\hline 15 & Male & Prefers not to say & iPhone 7 \\
\hline 16 & Male & 18 to 24 & iPhone $\mathrm{x}$ \\
\hline 17 & Male & 25 to 34 & iPhone 6s \\
\hline 18 & Male & 45 to 54 & iPhone 7 \\
\hline 19 & Male & 55 to 64 & iPhone 7 plus \\
\hline 20 & Male & 25 to 34 & iPhone 7 \\
\hline 21 & Male & 25 to 34 & iPhone 7 \\
\hline 22 & Female & 18 to 24 & iPhone 6 s plus \\
\hline 23 & Male & 25 to 24 & iPhone 7 \\
\hline 24 & Male & 35 to 44 & iPhone 6s \\
\hline 25 & Female & 45 to 54 & iPhone 7 plus \\
\hline 26 & Female & Prefers not to say & iPhone 7 \\
\hline
\end{tabular}

\section{RESULTS}

\section{A. Effectiveness}

Fig. 1 demonstrates the effectiveness performance percentage for each potential user for each session. 18 users had $100 \%$ correct completion rate over the three sessions. Users 2, 5, 13 and 14 showed positive progress across sessions, as they scored $92.85 \%$ at session one and then $100 \%$ by the third session. The correct completion tion rate of users 7, 17 and 19 remained constant over the three sessions at $92.85 \%$. Only user 26 showed negative progress across sessions, scoring $100 \%$ at both sessions 1 and 2 and then decreasing to $92.85 \%$ by the third session.

Consequently, as it is shown in Fig. 2 the effectiveness performance average increased over sessions. It started at $98.07 \%$, then increased to $98.62 \%$ and finally reached $98.90 \%$. 


\section{Effectiveness Performance}

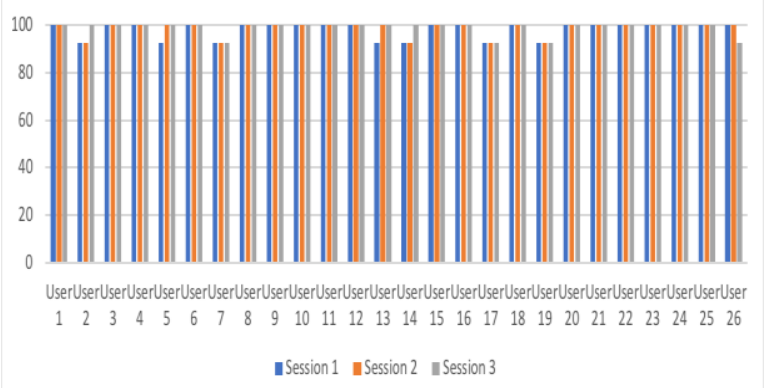

Fig. 1. Effectiveness Results.

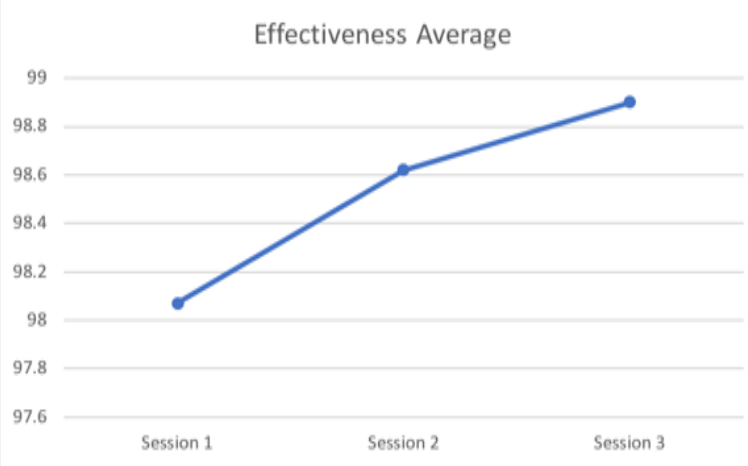

Fig. 2. Effectiveness Average.

\section{B. Efficiency}

1) Overall relative efficiency: Fig. 3 describes each task's overall relative efficiency percentage over the three sessions. All tasks except tasks 11 and 12 scored $100 \%$ in all three sessions. The overall relative efficiency percentage of tasks 11 and 12 improved over the sessions. Task 11's overall relative efficiency percentage was $97.15 \%$ in session 1, which increased to $100 \%$ in session 2 but decreased to $97.23 \%$ by the third session. Task 12's overall relative efficiency percentage started at $67.16 \%$ in session 1 , then increased to $74.3 \%$ in session 2 and slightly rose again to reach $79.8 \%$ in session 3 .

Fig. 4 shows the average percentage for the overall relative efficiency which is improved across the three sessions. It started at $97.45 \%$, then increased to $98.16 \%$ and finally reached $98.35 \%$.

\section{Overall Relative Efficiency}

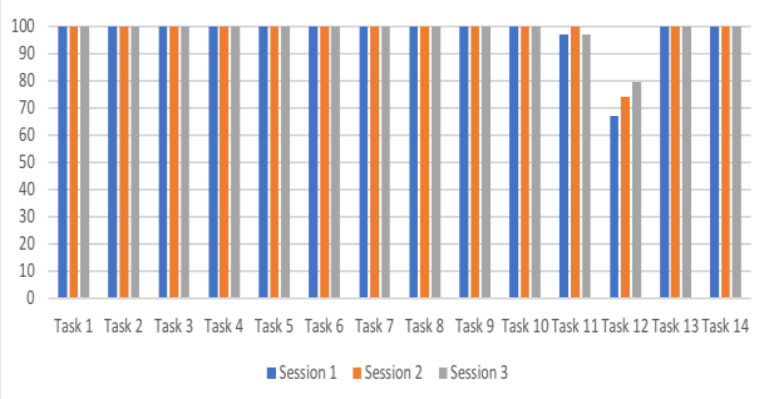

Fig. 3. Efficiency Results.

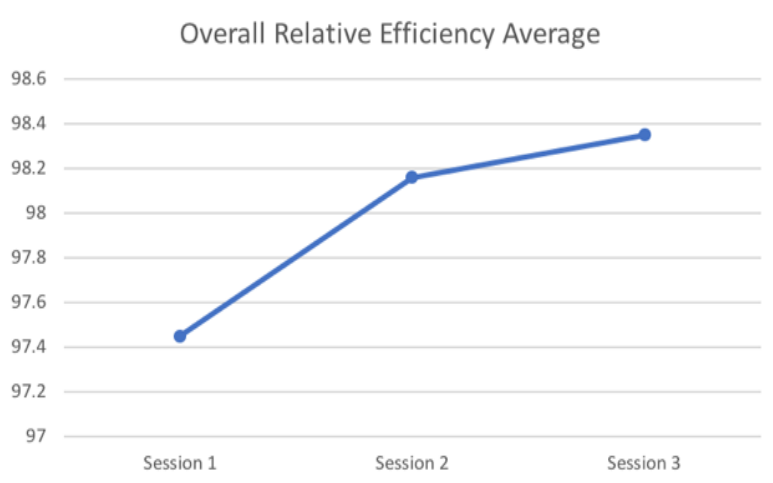

Fig. 4. Overall Relative Efficiency Average

2) Overall relative efficiency: Fig. 5 shows each task's time-based efficiency score over the three sessions. Task number 11 scored the highest time-based efficiency score out of the tasks. It started at 11.95 goals per second, then rose to 22.87 goals per second and, in the final session, reached 39.56 goals per second. Tasks 14, 8 and 4 scored the second, third and fourth highest time-based efficiency respectively. In contrast, task 1 scored the lowest time-based efficiency followed by tasks 12 and task 5 .

Fig. 6 presents the average score for the time-based efficiency which is improved over sessions. It started at 4.99 goals per second, then rose to 9.99 goals per second and, by session three, it had increased to 14.35 goals per second.

\section{Time-Based Efficiency}

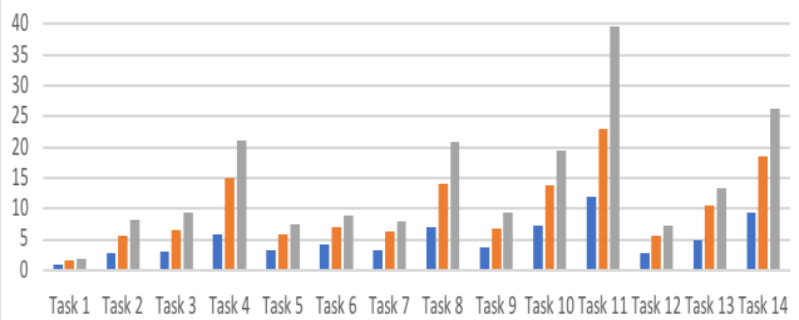

-Session 1 -Session 2 แSession 3

Fig. 5. Time-based Efficiency.

Time-Based Efficiency Average

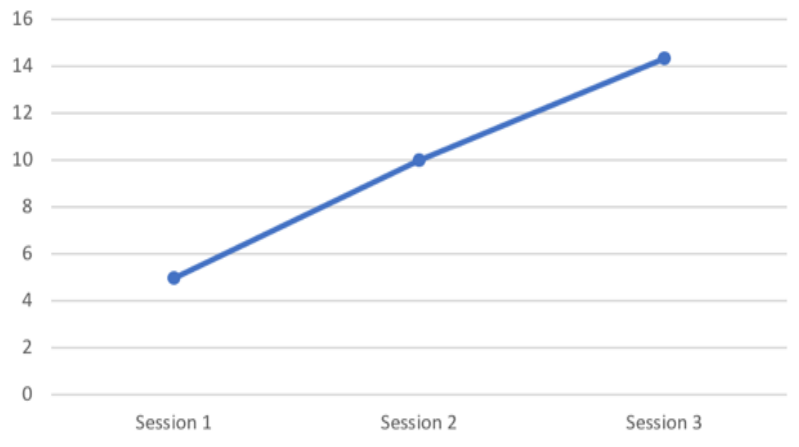

Fig. 6. Time-based Efficiency Average. 


\section{Satisfaction}

On average and as it is shown in Fig. 7, all the potential users found performing and completing the tasks easy. Using a scale where 1 is very easy and 7 is very difficult, user 14 scoured the highest value at 2.42 , followed by user 12 at 2.35 . However, six users, (users 6, 8, 9, 15, 23 and 25) found performing the tasks really easy as they all scored the lowest value of 1 .

\section{Cognitive Load}

Fig. 8 presents each subscale score in the cognitive load for each potential user. The cognitive load of users 11 and 21 was the most consistent, as both users had a score of 5 for all of the subscales. User 23's cognitive loading was the second most consistent, with scores between physical, temporal demand and frustration at 5\% and mental demand, performance and effort at $10 \%$. The cognitive loading of users 18 and 20 was not consistent, as the gap between all of the subscales scores was high. The highest score for all potential users was for mental demand and the lowest scores were for performance, frustration and physical demand.

Fig. 9 shows the total score for the cognitive load for each user. User 7 had the highest percentage at $51.3 \%$. The lowest percentage was for users 11 and 21 at $5 \%$, followed by user 23 at $8.3 \%$, user 12 at $9.6 \%$ and user 16 at $12.3 \%$.

\section{E. Errors}

Fig. 10 demonstrates each user's number of errors made over the sessions. Users 2, 5, 7, 13, 14, 17, 19 and 26 made errors while performing the tasks. The error rates of users 2, 5, 13 and 14 started with one error each in session 1 but had decreased to 0 by the third session. The error rate of users 7 , 17 and 19 was 1 in all the three sessions. User 26's error rate was 0 in both sessions 1 and 2 but one in session 3 .

Fig. 11 presents the total number of errors made by potential users which is decreased across sessions. It started with seven errors in session 1 , decreased to five errors in session 2 and four errors in session 3.

\section{Average Satisfaction Score}

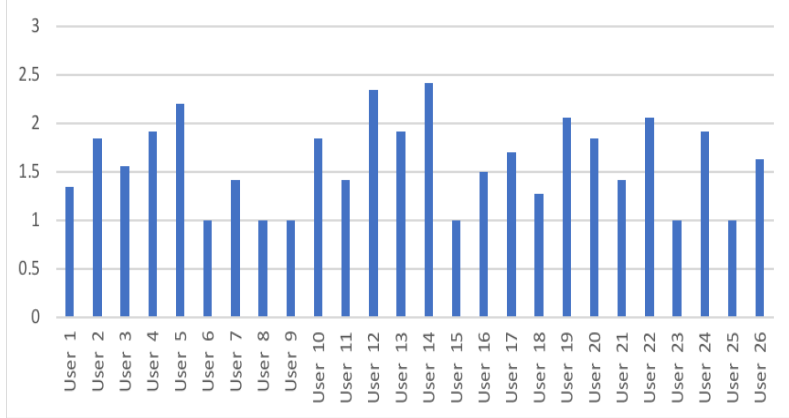

Fig. 7. Satisfaction Results.

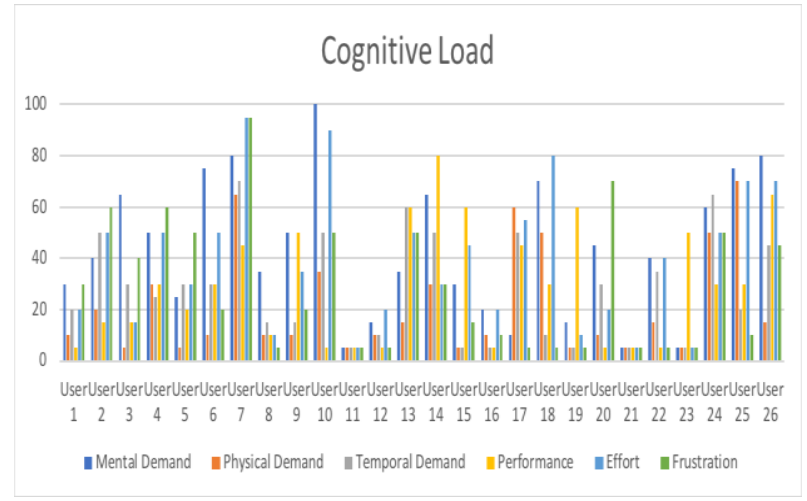

Fig. 8. Cognitive Load Results.

\section{Total Scoure for Cognitive Load}

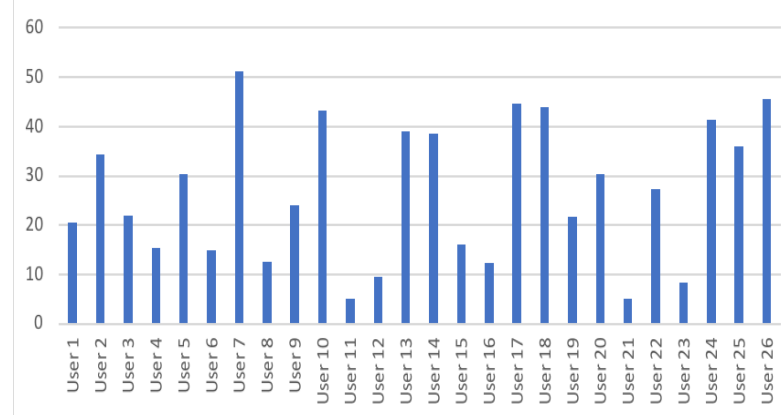

Fig. 9. Twazon's Screens for Ading Meal.

\section{Number of Errors}

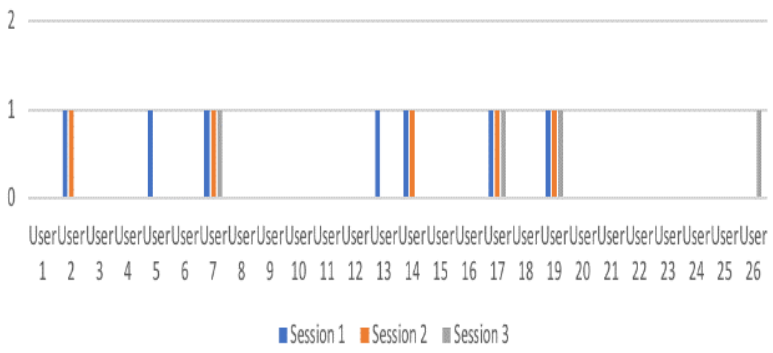

Fig. 10. Number of Errors.

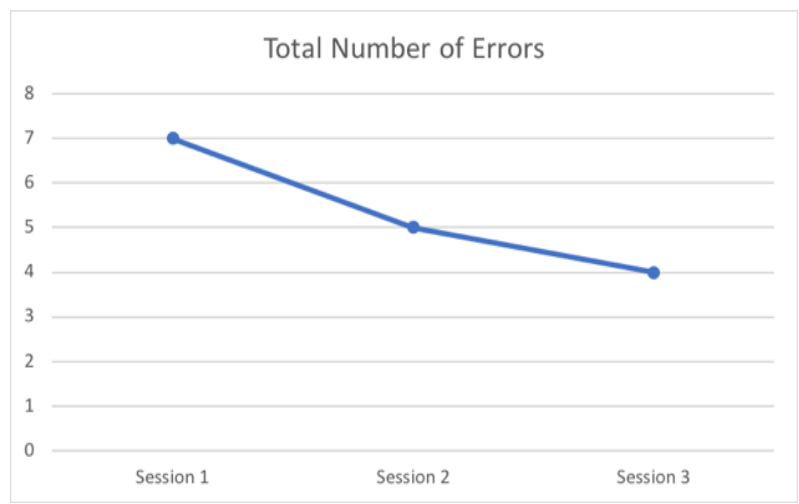

Fig. 11. Total Number of Errors. 


\section{USABILITY LEVEL BETWEEN USERS}

The results found that app's level of usability is different amongst potential users as users have diverse learning styles and technological literacy. Users 5 and 13 made mistakes only in the first session whereas users 2 and 14 made mistakes in both the first and second session. User 26 made her only mistake in the third session. Users 7,17 and 19 are only the users who made mistakes in all the three sessions. Moreover, user 14's average satisfaction score was the highest comparing to other users at 2.42 out of 7 . The total score of the cognitive load for user 7 was the highest amongst users at $51.3 \%$. Such differences between users is investigated by examining their video records from the usability testing and identifying the specific tasks in which they were not able to either perform correctly or complete. Considering their feedback from the recorded interviews, especially what they did not like about the app and their suggestions for improvement, is another compulsory step in the app development process.

\section{USABILITY ISSUES AND SOLUTIONS}

\section{A. Deep Navigation}

One potential user faced difficulty when asked to locate the option for learning how to correctly read and understand labels on food products. Such an issue affects the app's usability, as users might need to keep navigating to find what they are looking for and thus increasing both the time and steps taken to locate it. The food label option in the beta version of the app is four levels below the Home screen and users need to navigate the following levels to reach it:

- Home screen

- Setting

- Useful information

- Food

- Read food label

This issue is addressed by enhancing the navigation for the food label option. Now, it is located just two levels below the Home screen, as follows:

- Home screen

- Food

- Read food label

Due to this change, users will be required to perform fewer steps to find the food label option, thus decreasing the time needed compared to the previous design.

\section{B. Option Location}

The second usability issue is the location for updating users' information option compared to other options' location within the same screen. Fig. 12 shows the old design for the settings screen where the option for the personal information is located on the top left corner of the screen. While analyzing the video records for the usability testing and as the screenshot that is presented in Fig. 12, we found that six users did not recognize this option the first time they used the app and they were trying other options within the same screen.

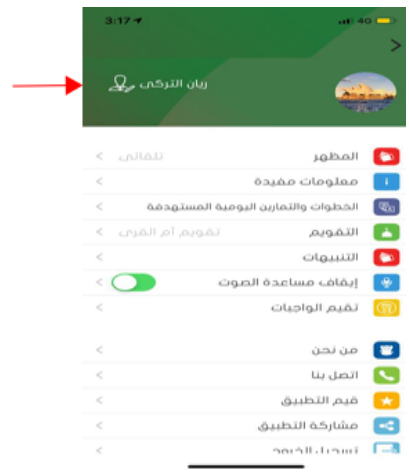

Fig. 12. Original Design for the Setting Screen.

This issue was addressed by changing the location of the personal information option so that it is located with the other options as it can be seen in Fig. 13. Through this change, it is much easier to recognize the option as users only now need to look at one place.

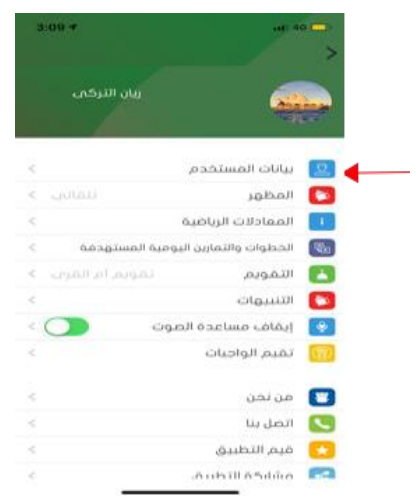

Fig. 13. New Design for the Setting Screen.

\section{SUGGESTIONS}

A few potential users suggested that it will be better for future users to have the option to use the app without the need to go through the registration process. Based on their recommendation, a new button that is labelled 'Try me' is added within the first screen of the app. Fig. 14 shows both the original and new design for the first screen. When users click on it, they will pass the registration process and start using the app. Then if they decide to continue using the app, they can input their information via the personal information option within the setting screen.

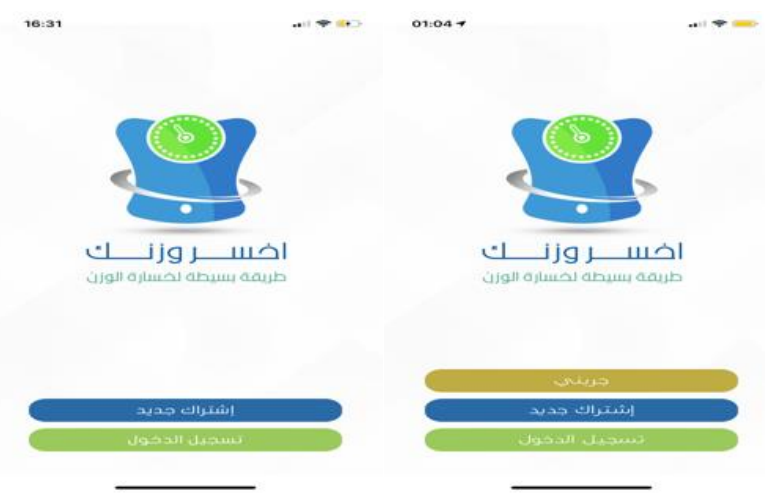

Fig. 14. Original and New Design for the First Screen. 


\section{CONCLUSION}

The analyses of the previous usability testing of two Arabic weight loss apps (Twazon and Aded Surat) showed that their level of usability is low, as a result users reported that they had difficulty using the apps. In this paper, we have improved and analyzed the usability testing of the Akser Waznk app. The usability of this app was improved over the three sessions. The percentage score for potential users' effectiveness enhanced between session one and session two and it further enhanced from session two to session three. Furthermore, the total number of errors decreased across the three sessions. Based on the observed increase of scores, the usage of the Akser Waznk app is easy to be learned and remembered which means that the app positively considers learnability and memorability attributes. Moreover, the majority of potential users recorded a low ratio in the satisfaction questionnaire. This is positive because a low value means that the app is very easy to be used. Additionally, the total score for cognitive load was not that high; only one potential user scored $75.6 \%$ which was the highest. This states that the majority of potential users had the ability to complete tasks correctly when performing other actions, such as speaking to the examiners.

\section{ACKNOWLEDGMENT}

This research is approved by the University of Technology Sydney, Human Research Ethics Committee (UTS HREC) approval number: ETH16-0833. The authors would like to thank all the potential users from the Armed Forces Hospitals in Taif, Saudi Arabia for their contribution within the usability testing.

\section{REFERENCES}

[1] Alturki, R.; Gay, V. Augmented and Virtual Reality in Mobile Fitness Applications: A Survey. In Applications of Intelligent Technologies in Healthcare, Springer: 2019; pp. 67-75.

[2] Sjöström, L.V. Mortality of severely obese subjects. The american journal of clinical nutrition 1992, 55, 516S-523S

[3] Alturki, R.; Gay, V. A Systematic Review on What Features should be supported by fitness Apps and Wearable to help users overcome obesity. IJRET: International Journal of Research in Engineering and Technology 2016, 5, 197-206.

[4] Alturki, R.; Gay, V. The Development of an Arabic Weight-Loss App Akser Waznk: Qualitative Results. JMIR formative research 2019, 3, e11785.

[5] Conroy, D.E.; Yang, C.-H.; Maher, J.P. Behavior change techniques in top-ranked mobile apps for physical activity. American journal of preventive medicine 2014, 46, 649-652.

[6] Arteaga, S.M.; Kudeki, M.; Woodworth, A.; Kurniawan, S. Mobile system to motivate teenagers' physical activity. In Proceedings of Proceedings of the 9th International Conference on Interaction Design and Children; pp. 1-10.
[7] Elkhodr, M.; Alsinglawi, B.; Alshehri, M. A Privacy Risk Assessment for the Internet of Things in Healthcare. In Applications of Intelligent Technologies in Healthcare, Springer: 2019; pp. 47-54.

[8] European Commission. Regulation (Eu) 2016/679 of the European Parliament and of the Council on the protection of natural persons with regard to the processing of personal data and on the free movement of such data, and repealing Directive 95/46/EC (General Data Protection Regulation). Available online: https://eur-lex.europa.eu/legalcontent/EN/TXT/?uri=celex\%3A32016 R0679 (accessed on 21 March 2020).

[9] Alshehri, M.D.; Hussain, F.K. A comparative analysis of scalable and context-aware trust management approaches for internet of things. In Proceedings of International conference on neural information processing; pp. 596-605.

[10] European Commission. Privacy Code of Conduct on mobile health apps. Available online: https://ec.europa.eu/digital-single-market/en/privacycode-conduct-mobile-health-apps (accessed on 19 March 2020).

[11] Alshehri, M.D.; Hussain, F.K.; Hussain, O.K. Clustering-driven intelligent trust management methodology for the internet of things (CITM-IoT). Mobile networks and applications 2018, 23, 419-431.)

[12] Olesenko, V.; Beklemysheva, A. Mobile Application Security: Best Practices for App Developers. Availabe online: https://steelkiwi.com /blog/mobile-application-security-best-practices-for-app-developers/ (accessed on 13 December 2019).

[13] Alshehri, M.D.; Hussain, F.K. A fuzzy security protocol for trust management in the internet of things (Fuzzy-IoT). Computing 2019, 101, 791-818.

[14] Alshehri, M.D.; Hussain, F.K. A Centralized Trust Management Mechanism for the Internet of Things (CTM-IoT). In Proceedings of 12th International Conference on Broadband and Wireless Computing, Communication and Applications (BWCCA-2017), Barcelona, Spain; pp. 533-543.

[15] Alshehri, M.D.; Hussain, F.; Elkhodr, M.; Alsinglawi, B.S. A Distributed Trust Management Model for the Internet of Things (DTMIoT). In Recent Trends and Advances in Wireless and IoT-enabled Networks, Springer: 2019; pp. 1-9.

[16] Bevan, N.; Carter, J.; Earthy, J.; Geis, T.; Harker, S. New ISO standards for usability, usability reports and usability measures. In Proceedings of International Conference on Human-Computer Interaction; pp. 268-278.

[17] Alturki, R.; Gay, V. Usability Attributes for Mobile Applications: A Systematic Review. In Recent Trends and Advances in Wireless and IoT-enabled Networks, Springer: 2019; pp. 53-62.

[18] Harrison, R.; Flood, D.; Duce, D. Usability of mobile applications: literature review and rationale for a new usability model. Journal of Interaction Science 2013, 1, 1-16.

[19] Alturki, R.; Gay, V. Usability Testing of Fitness Mobile Application: Methodology and Quantitative Results. In Proceedings of 7th International Conference on Computer Science, Engineering \& Applications (ICCSEA 2017), Copenhagen, Denmark; pp. 97-114.

[20] Alturki, R.; Gay, V. Usability Testing of Fitness Mobile Application: Case Study Aded Surat App. International Journal of Computer Science ad Information Technology (IJCSIT) 2017, 9, 107-127.

[21] Nielsen, J. Usability engineering; Elsevier: 1994.

[22] Rubin, J. Handbook of usability testing: how to plan, design, and conduct effective tests. Wiley technical communication library Show all parts in this series 1994.

[23] Dumas, J.S.; Redish, J. A practical guide to usability testing; Intellect books: 1999. 